\title{
Identification of the Rotor Time Constant in Induction Machines without Speed Sensor
}

\author{
M. Li*, J.N. Chiasson*, M. Bodson**, L.M. Tolbert* \\ *The University of Tennessee, ECE Department, Knoxville, USA \\ **The University of Utah, ECE Department, Salt Lake City, USA
}

\begin{abstract}
A differential-algebraic method is used to estimate the rotor time constant $T_{R}$ of an induction motor without measurements of the rotor speed/position. The method consists of solving for the roots of a polynomial equation in $T_{R}$ whose coefficients depend only on the stator currents, stator voltages, and their derivatives. Experimental results are presented.
\end{abstract}

Index Terms-Rotor Time Constant, Sensorless Speed Observer, Induction Motor.

\section{INTRODUCTION}

Induction motors are very attractive in many applications owing to their simple structure, low cost, and robust construction. Field-oriented control is now used to obtain high performance drive of the induction motor because it gives control characteristics similar to separately excited DC motors. Implementation of a (rotor-flux) field-oriented controller requires knowledge of the rotor speed and the rotor time constant $T_{R}$ to estimate the rotor flux linkages. There has been considerable work done in the last several years to implement a field-oriented controller without the use of a speed sensor [1][2][3][4][5][6]. However, many of these methods still require the value of $T_{R}$, which can change with time due to ohmic heating; that is, to be able to update the value of $T_{R}$ to the controller as it changes is valuable. The work presented here uses an algebraic approach to identify the rotor time constant $T_{R}$ without the motor speed information. It is most closely related to the ideas described in [7][8][9][10][11]. Specifically, it is shown that $T_{R}$ satisfies a polynomial equation whose coefficients are functions of the stator currents, the stator voltages, and their derivatives. A zero of this polynomial is the value of $T_{R}$. It is further shown $T_{R}$ is not identifiable by this technique under steady-state conditions. It is also true (and shown here) that a standard least-squares approach cannot identify $T_{R}$ under steady-state conditions. In [4], the speed $\omega$ and $T_{R}$ are identified assuming constant speed but not (sinusoidal) steady state. In [12], the speed is assumed constant, but the flux magnitude is perturbed by a small amplitude sinusoidal signal to identify $T_{R}$.

The paper is organized as follows. Section II introduces a space vector model of the induction motor. Section III uses this model to develop a differential-algebraic equation that $T_{R}$ must satisfy. Section IV shows that in steady state, $T_{R}$ is not identifiable by either the differential-algebraic method nor a standard linear least-squares method. Section V presents the experimental results, while Section VI gives the conclusions and future work.

\section{Mathematical Model of Induction Motor}

The starting point of the analysis is a space vector model of the induction motor given by (see e.g., pp. 568 of [13])

$$
\begin{aligned}
\frac{d}{d t} \underline{i}_{S} & =\frac{\beta}{T_{R}}\left(1-j n_{P} \omega T_{R}\right) \underline{\psi}_{R}-\gamma \underline{i}_{S}+\frac{1}{\sigma L_{S}} \underline{u}_{S} \\
\frac{d}{d t} \underline{\psi}_{R} & =-\frac{1}{T_{R}}\left(1-j n_{P} \omega T_{R}\right) \underline{\psi}_{R}+\frac{M}{T_{R}} \underline{i}_{S} \\
\frac{d \omega}{d t} & =\frac{n_{p} M}{J L_{R}} \operatorname{Im}\left\{\underline{i}_{S} \underline{\psi}_{R}^{*}\right\}-\frac{\tau_{L}}{J},
\end{aligned}
$$

where $\underline{i}_{S} \triangleq i_{S a}+j i_{S b}, \underline{\psi}_{R} \triangleq \psi_{R a}+j \psi_{R b}$, and $\underline{u}_{S} \triangleq u_{S a}+$ $j u_{S b}$. Here, $\theta$ is the position of the rotor, $\omega=d \theta / d t$ is the rotor speed, $n_{p}$ is the number of pole pairs, $i_{S a}, i_{S b}$ are the (two-phase equivalent) stator currents, $\psi_{R a}, \psi_{R b}$ are the (twophase equivalent) rotor flux linkages, $R_{S}, R_{R}$ are the stator and rotor resistances, respectively, $M$ is the mutual inductance, $L_{S}$ and $L_{R}$ are the stator and rotor inductances, respectively, $J$ is the moment of inertia of the rotor, and $\tau_{L}$ is the load torque. The symbols $T_{R}=\frac{L_{R}}{R_{R}}, \sigma=1-\frac{M^{2}}{L_{S} L_{R}}, \beta=\frac{M}{\sigma L_{S} L_{R}}$, $\gamma=\frac{R_{S}}{\sigma L_{S}}+\frac{\beta M}{T_{R}}$ have been used to simplify the expressions. $T_{R}$ is referred to as the rotor time constant, while $\sigma$ is called the total leakage factor.

\section{Differential-Algebraic ApproAch to $T_{R}$ ESTIMATION}

The idea of the differential-algebraic approach is to solve (1) and (2) for $T_{R}$ [14][15]. However, equations (1) and (2) are only four equations while there are six unknowns, namely $\psi_{R a}, \psi_{R b}, d \psi_{R a} / d t, d \psi_{R b} / d t, \omega$, and $T_{R}$. Equation (3) is not used because it introduces the additional unknown $\tau_{L}$. To find two more independent equations, equation (1) is differentiated to obtain

$$
\begin{aligned}
\frac{d^{2}}{d t^{2}} \underline{i}_{S}= & \frac{\beta}{T_{R}}\left(1-j n_{P} \omega T_{R}\right) \frac{d}{d t} \underline{\psi}_{R}-j n_{P} \beta \underline{\psi}_{R} \frac{d \omega}{d t} \\
& -\gamma \frac{d}{d t} \underline{i}_{S}+\frac{1}{\sigma L_{S}} \frac{d}{d t} \underline{u}_{S} .
\end{aligned}
$$

Using the (complex-valued) equations (1) and (2), one can solve for $\underline{\psi}_{R}$ and $\frac{d}{d t} \underline{\psi}_{R}$ in terms of $\omega, \underline{i}_{S}$ and $\underline{u}_{S}$ and substitute 
the resulting expressions into (4) to obtain

$$
\begin{aligned}
\frac{d^{2}}{d t^{2}} \underline{i}_{S}= & -\frac{1}{T_{R}}\left(1-j n_{P} \omega T_{R}\right)\left(\frac{d}{d t} \underline{i}_{S}+\gamma \underline{i}_{S}-\frac{1}{\sigma L_{S}} \underline{u}_{S}\right) \\
& +\frac{\beta M}{T_{R}^{2}}\left(1-j n_{P} \omega T_{R}\right) \underline{i}_{S}-\gamma \frac{d}{d t} \underline{i}_{S}+\frac{1}{\sigma L_{S}} \frac{d}{d t} \underline{u}_{S} \\
& -\frac{j n_{P} T_{R}}{1-j n_{P} \omega T_{R}}\left(\frac{d}{d t} \underline{i}_{S}+\gamma \underline{i}_{S}-\frac{1}{\sigma L_{S}} \underline{u}_{S}\right) \frac{d \omega}{d t} .
\end{aligned}
$$

Solving (5) for $d \omega / d t$ gives

$$
\begin{aligned}
& \frac{d \omega}{d t}=-\frac{\left(1-j n_{P} \omega T_{R}\right)^{2}}{j n_{P} T_{R}^{2}}+\frac{1-j n_{P} \omega T_{R}}{j n_{P} T_{R}} \times \\
& \frac{\frac{\beta M}{T_{R}^{2}}\left(1-j n_{P} \omega T_{R}\right) \underline{i}_{S}-\gamma \frac{d}{d t} \underline{i}_{S}+\frac{1}{\sigma L_{S}} \frac{d}{d t} \underline{u}_{S}-\frac{d^{2}}{d t^{2}} \underline{i}_{S}}{\frac{d}{d t} \underline{i}_{S}+\gamma \underline{i}_{S}-\frac{1}{\sigma L_{S}} \underline{u}_{S}} .
\end{aligned}
$$

The left-hand side of (6) is real, so the right-hand side must also be real. Note by (1) that $d \underline{i}_{S} / d t+\gamma \underline{i}_{S}-\underline{u}_{S} /\left(\sigma L_{S}\right)=$ $\frac{\beta}{T_{R}}\left(1-j n_{P} \omega T_{R}\right) \underline{\psi}_{R}$ so that the right-hand side of (6) is singular if and only if $\left|\underline{\psi}_{R}\right|=0$. Other than at startup, $\left|\underline{\psi}_{R}\right| \neq 0$ in normal operation of the motor. Separating the right-hand side of (6) into its real and imaginary parts, the real part has the form

$$
\begin{aligned}
\frac{d \omega}{d t}= & a_{2}\left(u_{S a}, u_{S b}, i_{S a}, i_{S b}\right) \omega^{2}+a_{1}\left(u_{S a}, u_{S b}, i_{S a}, i_{S b}\right) \omega \\
& +a_{0}\left(u_{S a}, u_{S b}, i_{S a}, i_{S b}\right) .
\end{aligned}
$$

The expressions for $a_{2}\left(u_{S a}, u_{S b}, i_{S a}, i_{S b}\right), a_{1}\left(u_{S a}, u_{S b}, i_{S a}\right.$, $\left.i_{S b}\right)$, and $a_{0}\left(u_{S a}, u_{S b}, i_{S a}, i_{S b}\right)$ are lengthy in terms of $u_{S a}$, $u_{S b}, i_{S a}, i_{S b}$, and their derivatives as well as of the machine parameters including $T_{R}$. As a consequence, they are not explicitly presented here. Their steady-state expressions are given in [6].

On the other hand, the imaginary part of the right-hand side of (6) must be zero. In fact, the imaginary part of (6) is a second degree polynomial equation in $\omega$ of the form

$$
\begin{aligned}
q(\omega) \triangleq \quad & q_{2}\left(u_{S a}, u_{S b}, i_{S a}, i_{S b}\right) \omega^{2}+q_{1}\left(u_{S a}, u_{S b}, i_{S a}, i_{S b}\right) \omega \\
& +q_{0}\left(u_{S a}, u_{S b}, i_{S a}, i_{S b}\right)
\end{aligned}
$$

and, if $\omega$ is the speed of the motor, then $q(\omega)=0$. The $q_{i}$ are functions of $u_{S a}, u_{S b}, i_{S a}, i_{S b}$, and their derivatives as well as of the machine parameters including $T_{R}$. The expressions for $q_{2}\left(u_{S a}, u_{S b}, i_{S a}, i_{S b}\right), q_{1}\left(u_{S a}, u_{S b}, i_{S a}, i_{S b}\right)$, and $q_{0}\left(u_{S a}, u_{S b}, i_{S a}, i_{S b}\right)$ are also lengthy and not explicitly presented here. (Their steady-state expressions are given in [6].) If the speed was measured, then (8) would be equal to zero and could then be solved for $T_{R}$. However, in the problem being considered, $\omega$ is not known. To eliminate $\omega, q(\omega)$ in (8) is differentiated to obtain

$$
\frac{d}{d t} q(\omega)=\left(2 q_{2} \omega+q_{1}\right) \frac{d \omega}{d t}+\dot{q}_{2} \omega^{2}+\dot{q}_{1} \omega+\dot{q}_{0}
$$

where $d q(\omega) / d t \equiv 0$ if $\omega$ is equal to the motor speed. Next, $d \omega / d t$ in (9) is replaced by the right-hand side of (7) so that
(9) may be written as

$$
\begin{aligned}
\frac{d q(\omega)}{d t}= & g(\omega) \triangleq 2 q_{2} a_{2} \omega^{3}+\left(2 q_{2} a_{1}+q_{1} a_{2}+\dot{q}_{2}\right) \omega^{2} \\
& +\left(2 q_{2} a_{0}+q_{1} a_{1}+\dot{q}_{1}\right) \omega+q_{1} a_{0}+\dot{q}_{0}
\end{aligned}
$$

$g(\omega)$ is a third-order polynomial equation in $\omega$ for which the speed of the motor is one of its zeros. Dividing ${ }^{1} g(\omega)$ in (10) by $q(\omega)^{2}$ in (8), $g(\omega)$ may be rewritten as

$$
\begin{aligned}
& g(\omega)=\frac{1}{q_{2}}\left(\left(2 q_{2} a_{2} \omega+2 q_{2} a_{1}-q_{1} a_{2}+\dot{q}_{2}\right) q(\omega)\right. \\
& \left.+r_{1}\left(u_{S a}, u_{S b}, i_{S a}, i_{S b}\right) \omega+r_{0}\left(u_{S a}, u_{S b}, i_{S a}, i_{S b}\right)\right)
\end{aligned}
$$

$$
\begin{aligned}
r_{1}\left(u_{S a}, u_{S b}, i_{S a}, i_{S b}\right) \triangleq \quad & 2 q_{2}^{2} a_{0}-q_{2} q_{1} a_{1}+q_{2} \dot{q}_{1}-2 q_{2} q_{0} a_{2} \\
& +q_{1}^{2} a_{2}-q_{1} \dot{q}_{2} \\
r_{0}\left(u_{S a}, u_{S b}, i_{S a}, i_{S b}\right) \triangleq \quad & q_{2} q_{1} a_{0}+q_{2} \dot{q}_{0}-2 q_{2} q_{0} a_{1} \\
& +q_{0} q_{1} a_{2}-q_{0} \dot{q}_{2} .
\end{aligned}
$$

If $\omega$ is equal to the speed of the motor, then both $g(\omega)=0$ and $q(\omega)=0$, and one obtains

$r(\omega) \triangleq r_{1}\left(u_{S a}, u_{S b}, i_{S a}, i_{S b}\right) \omega+r_{0}\left(u_{S a}, u_{S b}, i_{S a}, i_{S b}\right)=0$.

This is now a first-order polynomial equation in $\omega$ which uniquely determines the motor speed $\omega$ as long as $r_{1}$ (the coefficient of $\omega$ ) is nonzero. (It is shown in Appendix VII-A that $r_{1} \neq 0$ in steady state.) Solving for the motor speed $\omega$ using (14), one obtains

$$
\omega=-r_{0} / r_{1}
$$

Next, replace $\omega$ in (8) by the expression in (15) to obtain

$$
q_{2} r_{0}^{2}-q_{1} r_{0} r_{1}+q_{0} r_{1}^{2} \equiv 0 \text {. }
$$

The expressions for $q_{i}, r_{i}$ are in terms of motor parameters (including $T_{R}$ ) as well as the stator currents, voltages, and their derivatives. Expanding the expressions for $q_{0}, q_{1}, q_{2}, r_{0}$, and $r_{1}$, one obtains a twelfth-order polynomial equation in $T_{R}$, which can be written as

$$
\sum_{i=0}^{12} C_{i}\left(u_{S a}, u_{S b}, i_{S a}, i_{S b}\right) T_{R}^{i}=0 .
$$

Solving equation (17) gives $T_{R}$. The coefficients $C_{i}\left(u_{S a}, u_{S b}, i_{S a}, i_{S b}\right)$ of (17) contain third-order derivatives of the stator currents and second-order derivatives of the stator voltages making noise a concern. For short time intervals in which $T_{R}$ does not vary, (17) must hold identically with $T_{R}$ constant. In order to average out the effect of noise on the $C_{i},(17)$ is integrated over a time interval $\left[t_{1}, t_{2}\right]$ to obtain

$$
\sum_{i=0}^{12}\left(\frac{1}{t_{2}-t_{1}} \int_{t_{1}}^{t_{2}} C_{i}\left(u_{S a}, u_{S b}, i_{S a}, i_{S b}\right) d t\right) T_{R}^{i}=0 .
$$

${ }^{1}$ Given the polynomials $g(\omega), q(\omega)$ in $\omega$ with $\operatorname{deg}\{g(\omega)\}=$ $n_{g}, \operatorname{deg}\{q(\omega)\}=n_{q}$, the Euclidean division algorithm ensures that there are polynomials $\gamma(\omega), r(\omega)$ such that $g(\omega)=\gamma(\omega) q(\omega)+r(\omega)$ and $\operatorname{deg}\{r(\omega)\} \leq \operatorname{deg}\{q(\omega)\}-1=n_{q}-1$. Consequently if, for example, $\omega_{0}$ is a zero of both $g(\omega)$ and $q(\omega)$, then it must also be a zero of $r(\omega)$.

${ }^{2} q_{2} \neq 0$ if $\omega$ and the stator electrical frequency $\omega_{S}$ are nonzero, which hold under normal operating conditions. See [6][16]. 
There are 12 solutions satisfying (18). However, simulation results have always given 10 conjugate solutions. The remaining two solutions include the correct value of $T_{R}$ while the other one was either negative or close to zero. The method is to compute the coefficients $\frac{1}{t_{2}-t_{1}} \int_{t_{1}}^{t_{2}} C_{i} d t$ and then compute the roots of (18). Among the positive real roots is the correct value of $T_{R}$. Experimental results using this method are presented in Section V.

\section{Identifiability of $T_{R}$ IN Steady State}

\section{A. Differential-algebraic approach}

The polynomial (18) is now considered with the machine in steady state so that, in particular, the speed is constant. That is, $u_{S a}+j u_{S b}=\underline{U}_{S} e^{j \omega_{S} t}$ and $i_{S a}+j i_{S b}=\underline{I}_{S} e^{j \omega_{S} t}$ are substituted into (8) and (14). In steady state, the motor speed in (15) becomes (see Appendix VII-A and [16])

$$
\omega=-\frac{r_{0}}{r_{1}}=\frac{\omega_{S}(1-S)}{n_{p}}
$$

where $S \triangleq\left(\omega_{S}-n_{p} \omega\right) / \omega_{S}$ is the normalized slip and $\omega_{S}$ is the electrical frequency. Substituting the steady-state expressions for $q_{2}, q_{1}$, and $q_{0}$ as well as the expression (19) for $\omega$ into (8), one obtains $q_{2} \omega^{2}+q_{1} \omega+q_{0}=$

$$
\begin{aligned}
& \frac{n_{p}^{2} T_{R}^{2}\left|\underline{I}_{S}\right|^{4} \omega_{S}^{2} L_{S}(1-\sigma)^{2}(1-S)}{\sigma\left(1+S^{2} \omega_{S}^{2} T_{R}^{2}\right)}\left(\frac{\omega_{S}(1-S)}{n_{p}}\right)^{2} \\
& +\frac{n_{p} \omega_{S}\left|\underline{I}_{S}\right|^{4} L_{S}(1-\sigma)^{2}\left(1-\omega_{S}^{2} T_{R}^{2}(1-S)^{2}\right)}{\sigma\left(1+S^{2} \omega_{S}^{2} T_{R}^{2}\right)} \\
& \times\left(\frac{\omega_{S}(1-S)}{n_{p}}\right)-\frac{\left|\underline{I}_{S}\right|^{4} \omega_{S}^{2} L_{S}(1-\sigma)^{2}(1-S)}{\sigma\left(1+S^{2} \omega_{S}^{2} T_{R}^{2}\right)} \equiv 0 .
\end{aligned}
$$

That is, in steady state (8) and (14) hold independent of the value of $T_{R}$ and thus so does (17) making $T_{R}$ unidentifiable in steady state by this method.

\section{B. Linear least-squares approach}

Vélez-Reyes et al [3][4] have used least-squares methods for simultaneous parameter and speed identification in induction machines. In the approach used herein, $d \omega / d t$ is taken to be zero so that a linear (in the parameters) regressor model can be obtained. Specifically, consider the mathematical model of the induction motor in (5). Assuming constant speed, $d \omega / d t=0$ so that this equation reduces to

$$
\begin{aligned}
\frac{d^{2}}{d t^{2}} \underline{i}_{S}= & -\frac{1}{T_{R}}\left(1-j n_{P} \omega T_{R}\right)\left(\frac{d}{d t} \underline{i}_{S}+\gamma \underline{i}_{S}-\frac{1}{\sigma L_{S}} \underline{u}_{S}\right) \\
& +\frac{\beta M}{T_{R}^{2}}\left(1-j n_{P} \omega T_{R}\right) \underline{i}_{S}-\gamma \frac{d}{d t} \underline{i}_{S}+\frac{1}{\sigma L_{S}} \frac{d}{d t} \underline{u}_{S}
\end{aligned}
$$

where $\underline{i}_{S}=i_{S a}+j i_{S b}$ and $\underline{u}_{S}=u_{S a}+j u_{S b}$. Decomposing equation (20) into its real and imaginary parts gives

$$
\begin{aligned}
\frac{d^{2} i_{S a}}{d t}= & \frac{1}{T_{R}}\left(-\frac{d i_{S a}}{d t}-\frac{R_{S}}{\sigma L_{S}} i_{S a}+\frac{1}{\sigma L_{S}} u_{S a}\right) \\
& +n_{p} \omega\left(-\frac{d i_{S b}}{d t}-\frac{R_{S}}{\sigma L_{S}} i_{S b}+\frac{1}{\sigma L_{S}} u_{S b}\right) \\
& -\gamma \frac{d i_{S a}}{d t}+\frac{1}{\sigma L_{S}} \frac{d u_{S a}}{d t}
\end{aligned}
$$

and

$$
\begin{aligned}
\frac{d^{2} i_{S b}}{d t}= & \frac{1}{T_{R}}\left(-\frac{d i_{S b}}{d t}-\frac{R_{S}}{\sigma L_{S}} i_{S b}+\frac{1}{\sigma L_{S}} u_{S b}\right) \\
& -n_{p} \omega\left(-\frac{d i_{S a}}{d t}-\frac{R_{S}}{\sigma L_{S}} i_{S a}+\frac{1}{\sigma L_{S}} u_{S a}\right) \\
& -\gamma \frac{d i_{S b}}{d t}+\frac{1}{\sigma L_{S}} \frac{d u_{S b}}{d t} .
\end{aligned}
$$

The goal here is to estimate $T_{R}$ without knowledge of $\omega$. So, it is now assumed the motor parameters are all known except for $T_{R}$. The set of equations (21) and (22) may then be rewritten in regressor form as

$$
y(t)=W(t) K
$$

where $K \in \mathbb{R}^{2}, y \in \mathbb{R}^{2}$, and $W \in \mathbb{R}^{2 \times 2}$ are given by

$$
\begin{gathered}
K \triangleq\left[\begin{array}{c}
1 / T_{R} \\
n_{p} \omega
\end{array}\right], \\
y(t) \triangleq\left[\begin{array}{c}
\frac{d u_{S a}}{d t}-\sigma L_{S} \frac{d^{2} i_{S a}}{d t}-R_{S} \frac{d i_{S a}}{d t} \\
\frac{d u_{S b}}{d t}-\sigma L_{S} \frac{d^{2} i_{S b}}{d t}-R_{S} \frac{d i_{S b}}{d t}
\end{array}\right], \\
W(t) \triangleq\left[\begin{array}{cc}
L_{S} \frac{d i_{S a}}{d t}-u_{S a}+R_{S} i_{S a} & \sigma L_{S} \frac{d i_{S b}}{d t}-u_{S b}+R_{S} i_{S b} \\
L_{S} \frac{d i_{S b}}{d t}-u_{S b}+R_{S} i_{S b} & -\sigma L_{S} \frac{d i_{S a}}{d t}+u_{S a}-R_{S} i_{S a}
\end{array}\right] .
\end{gathered}
$$

The regressor system (23) is linear in the parameters. The standard linear least-squares approach is to let (i.e., collect data at) $t=0, T, 2 T, \cdots, N T$, multiply (23) on the left by $W^{T}(n T)$, sum $W^{T}(n T) y(n T)=W^{T}(n T) W(n T) K$ from $t=0$ to $t=N T$, and finally compute the solution to

$$
R_{W} K=R_{Y W}
$$

where

$R_{W} \triangleq \sum_{n=0}^{N} W^{T}(n T) W(n T), \quad R_{Y W} \triangleq \sum_{n=0}^{N} W^{T}(n T) y(n T)$.

A unique solution to (24) exists if and only if $R_{W}$ is invertible. However, $R_{W}$ is never invertible in steady state as is now shown. To proceed, define

$$
D(t)=\left[\begin{array}{rr}
i_{S b}(t) & -i_{S a}(t) \\
i_{S a}(t) & i_{S b}(t)
\end{array}\right] .
$$

In steady state where $u_{S a}+j u_{S b}=\underline{U}_{S} e^{j \omega_{S} t}$ and $i_{S a}+$ $j i_{S b}=\underline{I}_{S} e^{j \omega_{S} t}, \operatorname{det}(D(t))=i_{S a}^{2}(t)+i_{S b}^{2}(t)=\left|\underline{I}_{S}\right|^{2}$, $D(t)^{T} D(t)=\left|\underline{I}_{S}\right|^{2} I_{2 \times 2}$. Multiply both sides of (23) on the left by $D(t)$ to obtain

$$
D(t) y(t)=D(t) W(t) K
$$

or

$$
\begin{aligned}
& {\left[\begin{array}{c}
R_{S} \omega_{S}\left|\underline{I}_{S}\right|^{2}-\omega_{S} P \\
\sigma L_{S} \omega_{S}^{2}\left|\underline{I}_{S}\right|^{2}-\omega_{S} Q
\end{array}\right]=} \\
& {\left[\begin{array}{cc}
-\omega_{S} L_{S}\left|\underline{I}_{S}\right|^{2}+Q & R_{S}\left|\underline{I}_{S}\right|^{2}-P \\
R_{S}\left|\underline{I}_{S}\right|^{2}-P & \sigma L_{S} \omega_{S}\left|\underline{I}_{S}\right|^{2}-Q
\end{array}\right] K}
\end{aligned}
$$


where $P \triangleq u_{S a} i_{S a}+u_{S b} i_{S b}$ and $Q \triangleq u_{S b} i_{S a}-u_{S a} i_{S b}$ are the real and reactive powers, respectively, whose steady-state expressions are given by (30) and (31) in the Appendix. Using (30) and (31) to replace $P$ and $Q$ in (25), one obtains

$$
\begin{aligned}
\bar{D} & \triangleq D(t) W(t) \\
& =-\frac{\left|\underline{I}_{S}\right|^{2}(1-\sigma) \omega_{S} L_{S}}{1+S^{2} \omega_{S}^{2} T_{R}^{2}}\left[\begin{array}{cc}
S^{2} \omega_{S}^{2} T_{R}^{2} & S \omega_{S} T_{R} \\
S \omega_{S} T_{R} & 1
\end{array}\right] \\
\bar{Y} & \triangleq D(t) y(t) \\
& =-\omega_{S} \frac{|\underline{I}|^{2}(1-\sigma) \omega_{S} L_{S}}{1+S^{2} \omega_{S}^{2} T_{R}^{2}}\left[\begin{array}{c}
S \omega_{S} T_{R} \\
1
\end{array}\right]
\end{aligned}
$$

That is, in steady state, $\bar{D} \triangleq D(t) W(t) \in \mathbb{R}^{2 \times 2}$ and $\bar{Y} \triangleq$ $D(t) y(t) \in \mathbb{R}^{2}$ are constant matrices. Further, it is easily seen that the determinant of $\bar{D} \triangleq D(t) W(t)$ is zero. Also,

$$
\begin{aligned}
R_{D W} & \left.\triangleq \sum_{n=1}^{N}(D(n T) W(n T))^{T}(D(n T) W(n T))\right) \\
& =\left|\underline{I}_{S}\right|^{2} \sum_{n=1}^{N} W^{T}(n T) W(n T)=\left|\underline{I}_{S}\right|^{2} R_{W} .
\end{aligned}
$$

$R_{D W}$ is singular because $D(t) W(t)$ is constant and singular. It then follows that $R_{W}$ is also singular using steady-state data. Further,

$$
\begin{aligned}
R_{D W Y} & \left.\triangleq \sum_{n=1}^{N}(D(n T) W(n T))^{T}(D(n T) y(n T))\right) \\
& =\left|\underline{I}_{S}\right|^{2} \sum_{n=1}^{N} W^{T}(n T) y(n T)=\left|\underline{I}_{S}\right|^{2} R_{Y W} .
\end{aligned}
$$

Thus $R_{W}$ and $R_{Y W}$ are given by

$$
\begin{aligned}
R_{W} & =R_{D W} /\left|\underline{I}_{S}\right|^{2}=N \bar{D}^{T} \bar{D} /\left|\underline{I}_{S}\right|^{2} \\
& =\frac{N\left|\underline{I}_{S}\right|^{2}(1-\sigma)^{2} \omega_{S}^{2} L_{S}^{2}}{1+S^{2} \omega_{S}^{2} T_{R}^{2}}\left[\begin{array}{cc}
S^{2} \omega_{S}^{2} T_{R}^{2} & S \omega_{S} T_{R} \\
S \omega_{S} T_{R} & 1
\end{array}\right]
\end{aligned}
$$$$
R_{Y W}=R_{D W Y} /\left|\underline{I}_{S}\right|^{2}=N \bar{D}^{T} \bar{Y} /\left|\underline{I}_{S}\right|^{2}
$$$$
=\omega_{S} \frac{N\left|\underline{I}_{S}\right|^{2}(1-\sigma)^{2} \omega_{S}^{2} L_{S}^{2}}{1+S^{2} \omega_{S}^{2} T_{R}^{2}}\left[\begin{array}{c}
S \omega_{S} T_{R} \\
1
\end{array}\right] \text {, }
$$

where again $\bar{D}$ and $\bar{Y}$ are from (26) and (27), respectively.

By inspection of $(28)$ and $(29), K=\left[0 \omega_{S}\right]^{T}$ is one solution to $(24)$. The null space of $R_{W}$ is generated by $\left[-1 / T_{R} S \omega_{S}\right]^{T}$ so that all possible solutions are given by $\left[0 \omega_{S}\right]^{T}+\alpha\left[-1 / T_{R} S \omega_{S}\right]^{T}$ for some $\alpha \in \mathbb{R}$. In summary, solving (24) using steady-state data leads to an infinite set of solutions so that $T_{R}$ is not identifiable using the linear regressor (23) with steady-state data.

\section{EXPERIMENTAL RESUlTS}

To demonstrate the viability of the speed sensorless estimator (18) for $T_{R}$, experiments were performed. A three-phase, $0.5 \mathrm{hp}, 1735 \mathrm{rpm}\left(n_{p}=2\right.$ pole-pair) induction motor was driven by an ALLEN-BRADLEY PWM inverter to obtain the data. Given a speed command to the inverter, it produces PWM voltages to drive the induction motor to the commanded speed. Here a step speed command was chosen to bring the motor from standstill up to the rated speed of $188 \mathrm{rad} / \mathrm{s}$. The stator currents and voltages were sampled at $10 \mathrm{kHz}$. The real-time computing system RTLAB from OPAL-RT with a fully integrated hardware and software system was used to collect data [17]. Filtered differentiation (using digital filters) was used for the derivatives of the voltages and currents. Specifically, the signals were filtered with a third-order Butterworth filter whose cutoff frequency was $100 \mathrm{~Hz}$. The voltages and currents were put through a $3-2$ transformation to obtain their twophase equivalent values.

Using the data $\left\{u_{S a}, u_{S b}, i_{S a}, i_{S b}\right\}$ collected between $0.84 \mathrm{sec}$ to $0.91 \mathrm{sec}$, which includes the time the motor accelerates, the quantities $d u_{S a} / d t, d u_{S a} / d t, d i_{S a} / d t, d i_{S b} / d t$, $d^{2} i_{S a} / d t^{2}, d^{2} i_{S b} / d t^{2}, d^{3} i_{S a} / d t^{3}, d^{3} i_{S b} / d t^{3}$ are calculated and used to evaluate the coefficients $C_{i}, i=1,2, \cdots, 12$ in equation (18). Solving (18), one obtains the 12 solutions

$$
\begin{aligned}
T_{R 1} & =+0.1064 & & T_{R 2}=-0.0186 \\
T_{R 3} & =-0.0576+j 0.0593 & & T_{R 4}=-0.0576-j 0.0593 \\
T_{R 5} & =-0.0037+j 0.0166 & & T_{R 6}=-0.0037-j 0.0166 \\
T_{R 7} & =-0.0072+j 0.0103 & & T_{R 8}=-0.0072-j 0.0103 \\
T_{R 9} & =+0.0125+j 0.0077 & & T_{R 10}=+0.0125-j 0.0077 \\
T_{R 11} & =+0.0065+j 0.0018 & & T_{R 12}=+0.0065-j 0.0018 .
\end{aligned}
$$

$T_{R}$ must be a real positive number, so $T_{R}=0.1064$ is the only possible choice. This value compares favorably with the value of $T_{R}=0.11$ obtained using the method of Wang et al [18], which requires a speed sensor.

To illustrate the identified $T_{R}$, a simulation of the induction motor model was carried out using the measured voltages as input. Then the simulation's output [stator currents computed according to (1) and (2)] are used to compare with the measured (stator currents) outputs. Figure 1 shows the sampled two-phase equivalent current $i_{S b}$ and its simulated response $i_{S b-s i m}$. The phase $a$ current $i_{S a}$ is similar, but shifted by $\pi /\left(2 n_{p}\right)$. The resulting phase $b$ current $i_{S b-s i m}$ from the simulation corresponds well with the actual measured current $i_{S b}$. Note that in equation (1) $\gamma=\frac{R_{S}}{\sigma L_{S}}+\frac{\beta M}{T_{R}}$ also depends on $T_{R}$.

\section{CONCLUSIONS AND FUture WORK}

This paper presented a differential-algebraic approach to the estimation of the rotor time constant of an induction motor without using a speed sensor. The experimental results demonstrated the practical viability of this method. Though the method is not applicable in steady state, neither is a standard linear least-squares approach. Future work includes studying an on-line implementation of the estimation algorithm and using such an online estimate in a speed sensorless fieldoriented controller.

\section{Appendix: Steady-State ExPREssions}

In the following, $\omega_{S}$ denotes the stator frequency and $S$ denotes the normalized slip defined by $S \triangleq\left(\omega_{S}-n_{p} \omega\right) / \omega_{S}$. With $u_{S a}+j u_{S b}=\underline{U}_{S} e^{j \omega_{S} t}$ and $i_{S a}+j i_{S b}=\underline{I}_{S} e^{j \omega_{S} t}$, it is 


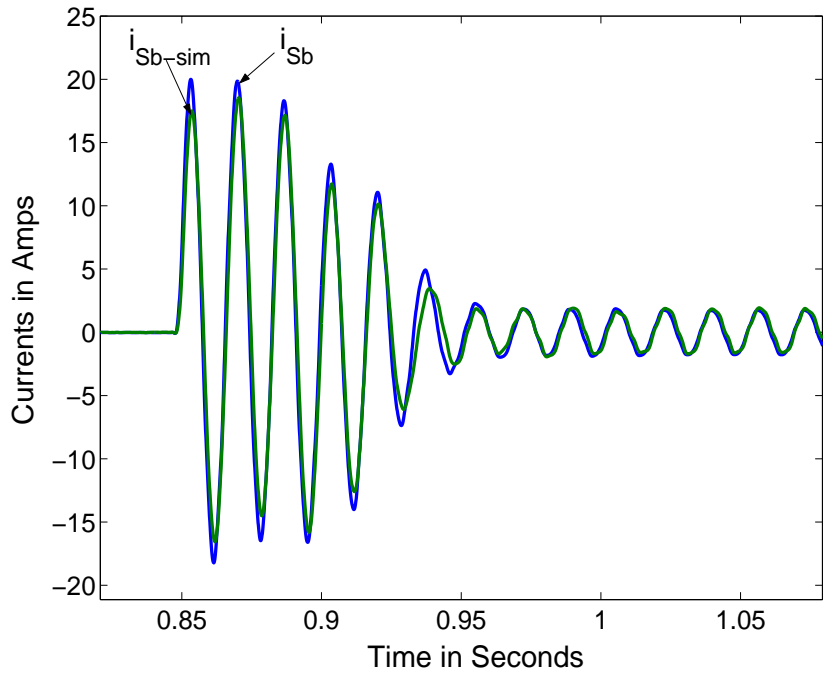

Fig. 1. Phase $b$ current $i_{S b}$ and its simulated response $i_{S b-s i m}$.

shown in [19] that under steady-state conditions, the complex phasors $\underline{U}_{S}$ and $\underline{I}_{S}$ are related by $\left(S_{p} \triangleq \frac{R_{R}}{\sigma \omega_{S} L_{R}}=\frac{1}{\sigma \omega_{S} T_{R}}\right)$

$$
\begin{aligned}
\underline{I}_{S} & =\frac{\underline{U}_{S}}{R_{S}+j \omega_{S} L_{S}\left(\left(1+j \frac{S}{S_{p}}\right) /\left(1+j \frac{S}{\sigma S_{p}}\right)\right)} \\
& =\frac{\underline{U}_{S}}{\left(R_{S}+\frac{(1-\sigma) S \omega_{S}^{2} L_{S} T_{R}}{1+S^{2} \omega_{S}^{2} T_{R}^{2}}\right)+j \frac{\omega_{S} L_{S}\left(1+\sigma S^{2} \omega_{S}^{2} T_{R}^{2}\right)}{1+S^{2} \omega_{S}^{2} T_{R}^{2}}},
\end{aligned}
$$

and straightforward calculations (see [6]) give

$$
\begin{aligned}
P & \triangleq u_{S a} i_{S a}+u_{S b} i_{S b}=R_{e}\left(\underline{U}_{S} \underline{I}_{S}^{*}\right) \\
& =\left|\underline{I}_{S}\right|^{2}\left(R_{S}+\frac{(1-\sigma) S \omega_{S}^{2} L_{S} T_{R}}{1+S^{2} \omega_{S}^{2} T_{R}^{2}}\right) \\
Q & \triangleq u_{S b} i_{S a}-u_{S a} i_{S b}=I_{m}\left(\underline{U}_{S} \underline{I}_{S}^{*}\right) \\
& =\left|\underline{I}_{S}\right|^{2} \frac{\omega_{S} L_{S}\left(1+\sigma S^{2} \omega_{S}^{2} T_{R}^{2}\right)}{1+S^{2} \omega_{S}^{2} T_{R}^{2}} .
\end{aligned}
$$

\section{A. Steady-State Expression for $r_{1}$ and $r_{0}$}

It is now shown that the steady-state value of $r_{1}$ in (12) is nonzero. Substituting the steady-state values of $q_{2}, q_{1}, q_{0}, a_{2}$, $a_{1}$, and $a_{0}$ shown in [6] (noting that $\dot{q}_{1} \equiv 0$ and $\dot{q}_{2} \equiv 0$ in steady state) into (12) gives

$$
\begin{aligned}
r_{1}= & -\left|\underline{I}_{S}\right|^{6}\left(\frac{1}{1+S^{2} \omega_{S}^{2} T_{R}^{2}}\right)^{3} \frac{n_{p}^{4}(1-\sigma)^{6} L_{S}^{2}}{\sigma^{4}} \times \\
& \omega_{S}^{3}\left(1+T_{R}^{2} \omega_{S}^{2}(1-S)^{2}\right)^{2} \frac{1}{d e n} \\
r_{0}= & \left|\underline{I}_{S}\right|^{6}\left(\frac{1}{1+S^{2} \omega_{S}^{2} T_{R}^{2}}\right)^{3} \frac{n_{p}^{3}(1-\sigma)^{6} L_{S}^{2}}{\sigma^{4}} \times \\
& \omega_{S}^{4}(1-S)\left(1+\omega_{S}^{2} T_{R}^{2} \times(1-S)^{2}\right)^{2} \frac{1}{d e n}
\end{aligned}
$$

where

$$
\begin{aligned}
\text { den } \triangleq & n_{p} T_{R}\left|\underline{I}_{S}\right|^{4}\left(\left(\frac{(1-\sigma)}{\sigma T_{R}} \frac{1+S^{2} \omega_{S}^{2} T_{R}^{2}-S \omega_{S}^{2} T_{R}^{2}}{1+S^{2} \omega_{S}^{2} T_{R}^{2}}\right)^{2}\right. \\
& \left.+\left(\frac{(1-\sigma)}{\sigma} \frac{\omega_{S}}{1+S^{2} \omega_{S}^{2} T_{R}^{2}}\right)^{2}\right) .
\end{aligned}
$$

Recall from Section III [following (6)] that den $=0$ if and only if $\left|\underline{\psi}_{R}\right|=0$. It is then seen that $r_{1} \neq 0$ in steady state.

\section{REFERENCES}

[1] K. Rajashekara, A. Kawamura, and K. Matsuse, Eds., Sensorless Control of AC Motor Drives - Speed and Position Sensorless Operation. IEEE Press, 1996.

[2] P. Vas, Sensorless Vector Control and Direct Torque Control. Oxford University Press, 1998.

[3] M. Vélez-Reyes, W. L. Fung, and J. E. Ramos-Torres, "Developing robust algorithms for speed and parameter estimation in induction machines," in Proceedings of the IEEE Conference on Decision and Control, 2001, pp. 2223-2228, orlando, Florida.

[4] M. Vélez-Reyes, "Decomposed algorithms for parameter estimation," Ph.D. dissertation, Massachusetts Institute of Technology, 1992.

[5] M. Bodson and J. Chiasson, "A comparison of sensorless speed estimation methods for induction motor control," in Proceedings of the 2002 American Control Conference, May 2002, pp. 3076-3081, anchorage, AK.

[6] M. Li, J. Chiasson, M. Bodson, and L. M. Tolbert, "Observability of speed in an induction motor from stator currents and voltages," in IEEE Conference on Decision and Control, December 2005, pp. 3438-3443, seville Spain.

[7] J. Reger, H. S. Ramirez, and M. Fliess, "On non-asymptotic observation of nonlinear systems," in Proceedings of the 44th IEEE Conference on Decision and Control, December 2005, pp. 4219-4224, seville Spain.

[8] M. Diop and M. Fliess, "On nonlinear observability," in Proceedings of the 1st European Control Conference. Hermès, Paris, 1991, pp. $152-157$.

[9] M. Fliess and H. Síra-Ramirez, "Control via state estimation of some nonlinear systems," in Symposium on Nonlinear Control Systems (NOLCOS-2004), September 2004, stuttgart, Germany.

[10] M. Fliess, C. Join, and H. Síra-Ramirez, "Complex continuous nonlinear systems: Their black box identification and their control," in 14th IFAC Symposium on System Identification (SISYD 2006), 2006, newcastle, Austalia.

[11] M. P. Saccomani, "Some results on parameter identification of nonlinear systems," Cardiovascular Engineering: An Internation Journal, vol. 4, no. 1, pp. 95-102, March 2004.

[12] I.-J. Ha and S.-H. Lee, "An online identification method for both stator and rotor resistances of induction motors without rotational transducers," IEEE Transactions on Industrial Electronics, vol. 47, no. 4, pp. 842-853, August 2000.

[13] J. Chiasson, Modeling and High-Performance Control of Electric Machines. John Wiley \& Sons, 2005.

[14] M. Diop and M. Fliess, "Nonlinear observability, identifiability and persistent trajectories," in IEEE Conference on Decision and Control. Brighton England, 1991, pp. 714-719.

[15] D. Nešić, I. M. Y. Mareels, S. T. Glad, and M. Jirstrand, "Software for control system analysis and design: symbol manipulation," in Encyclopedia of Electrical Engineering. John Wiley \& Sons, J. Webster, Editor, 2001, available online at http://www.interscience.wiley.com:83/eeee/.

[16] M. Li, "Differential-algebraic approach to speed and parameter estimation of the induction motor," Ph.D. dissertation, The Univesity of Tennessee, 2005.

[17] Opal-RT Technologies, "RT-LAB," see http://www.opal-rt.com.

[18] K. Wang, J. Chiasson, M. Bodson, and L. M. Tolbert, "An on-line rotor time constant estimator for the induction machine," in Proceedings of the IEEE International Electric Machines and Drives Conference, May 2005, pp. 608-614, san Antonio TX

[19] W. Leonhard, Control of Electrical Drives. 3rd Edition, SpringerVerlag, Berlin, 2001 